\title{
An extreme particle accelerator in the Galactic plane: HESS J1826-130
}

H. E. S. S. Collaboration: H. Abdalla ${ }^{1}$, R. Adam ${ }^{2}$, F. Aharonian ${ }^{3,4,5}$, F. Ait Benkhali ${ }^{4}$, E.O. Angüner ${ }^{6, \star}$, C. Arcaro $^{1}$, C. Armand ${ }^{7}$, T. Armstrong ${ }^{8}$, H. Ashkar ${ }^{9}$, M. Backes ${ }^{10,1}$, V. Baghmanyan ${ }^{11}$, V. Barbosa Martins ${ }^{12}$, A. Barnacka ${ }^{13}$, M. Barnard ${ }^{1}$, Y. Becherini ${ }^{14}$, D. Berge ${ }^{12}$, K. Bernlöhr ${ }^{4}$, B. Bi ${ }^{15}$, M. Böttcher ${ }^{1}$, C. Boisson ${ }^{16}$, J. Bolmont ${ }^{17}$, M. de Bony de Lavergne ${ }^{7}$, P. Bordas ${ }^{40, \star}$, M. Breuhaus ${ }^{4}$,

F. Brun ${ }^{9}$, P. Brun ${ }^{9}$, M. Bryan ${ }^{18}$, M. Büchele ${ }^{19}$, T. Bulik ${ }^{20}$, T. Bylund ${ }^{14}$, S. Caroff ${ }^{17}$, A. Carosi ${ }^{7}$, S. Casanova ${ }^{11,4, \star}$, T. Chand ${ }^{1}$,

S. Chandra ${ }^{1}$, A. Chen ${ }^{21}$, G. Cotter ${ }^{8}$, M. Curyło ${ }^{20}$, J. Damascene Mbarubucyeye ${ }^{12}$, I. D. Davids ${ }^{10}$, J. Davies ${ }^{8}$, C. Deil ${ }^{4}$,

J. Devin ${ }^{22}$, P. deWilt ${ }^{23}$, L. Dirson ${ }^{24}$, A. Djannati-Ataï ${ }^{25}$, A. Dmytriiev ${ }^{16}$, A. Donath ${ }^{4}$, V. Doroshenko ${ }^{15}$, C. Duffy ${ }^{26}$, J. Dyks $^{27}$,

K. Egberts $^{28}$, F. Eichhorn ${ }^{19}$, S. Einecke ${ }^{23}$, G. Emery ${ }^{17}$, J.-P. Ernenwein ${ }^{6}$, K. Feijen ${ }^{23}$, S. Fegan ${ }^{2}$, A. Fiasson ${ }^{7}$,

G. Fichet de Clairfontaine ${ }^{16}$, G. Fontaine ${ }^{2}$, S. Funk ${ }^{19}$, M. Füßling ${ }^{12}$, S. Gabici ${ }^{25}$, Y. A. Gallant ${ }^{29}$, G. Giavitto ${ }^{12}$, L. Giunti ${ }^{25,9}$,

D. Glawion ${ }^{30}$, J. F. Glicenstein ${ }^{9}$, D. Gottschall ${ }^{15}$, M.-H. Grondin ${ }^{22}$, J. Hahn ${ }^{4}$, M. Haupt ${ }^{12}$, G. Hermann ${ }^{4}$, J. A. Hinton ${ }^{4}$,

W. Hofmann ${ }^{4}$, C. Hoischen ${ }^{28}$, T. L. Holch ${ }^{31}$, M. Holler ${ }^{32}$, M. Hörbe ${ }^{8}$, D. Horns ${ }^{24}$, D. Huber ${ }^{32}$, M. Jamrozy ${ }^{13}$, D. Jankowsky ${ }^{19}$, F. Jankowsky ${ }^{30}$, A. Jardin-Blicq ${ }^{4}$, V. Joshi ${ }^{19}$, I. Jung-Richardt ${ }^{19}$, E. Kasai ${ }^{10}$, M. A. Kastendieck ${ }^{24}$, K. Katarzyński ${ }^{34}$, U. Katz ${ }^{19}$,

D. Khangulyan ${ }^{33}$, B. Khélifi ${ }^{25}$, S. Klepser ${ }^{12}$, W. Kluźniak ${ }^{27}$, Nu. Komin ${ }^{21}$, R. Konno ${ }^{12}$, K. Kosack ${ }^{9}$, D. Kostunin ${ }^{12}$, M. Kreter ${ }^{1}$,

G. Lamanna ${ }^{7}$, A. Lemière ${ }^{25}$, M. Lemoine-Goumard ${ }^{22}$, J.-P. Lenain ${ }^{17}$, C. Levy ${ }^{17}$, T. Lohse ${ }^{31}$, I. Lypova ${ }^{12}$, J. Mackey ${ }^{3}$, J. Majumdar ${ }^{12}$,

D. Malyshev ${ }^{15}$, D. Malyshev ${ }^{19}$, V. Marandon ${ }^{4}$, I. Marchegiani ${ }^{21}$, A. Marcowith ${ }^{29}$, A. Mares ${ }^{22}$, G. Martí-Devesa ${ }^{32}$, R. Marx $^{30,4}$,

G. Maurin $^{7}$, P. J. Meintjes ${ }^{35}$, M. Meyer ${ }^{19}$, A. Mitchell ${ }^{38,39}$, R. Moderski ${ }^{27}$, M. Mohamed ${ }^{30}$, L. Mohrmann ${ }^{19}$, A. Montanari ${ }^{9}$,

C. Moore ${ }^{26}$, P. Morris ${ }^{8}$, E. Moulin ${ }^{9}$, J. Muller $^{2}$, T. Murach ${ }^{12}$, K. Nakashima ${ }^{19}$, A. Nayerhoda ${ }^{11}$, M. de Naurois ${ }^{2}$, H. Ndiyavala ${ }^{1}$,

F. Niederwanger ${ }^{32}$, J. Niemiec ${ }^{11}$, L. Oakes ${ }^{31}$, P. O’Brien ${ }^{26}$, H. Odaka ${ }^{36}$, S. Ohm ${ }^{12}$, L. Olivera-Nieto ${ }^{4}$, E. de Ona Wilhelmi ${ }^{12}$,

M. Ostrowski ${ }^{13}$, I. Oya ${ }^{12 \star \star}$, M. Panter ${ }^{4}$, S. Panny ${ }^{32}$, R. D. Parsons ${ }^{31}$, G. Peron ${ }^{4}$, B. Peyaud ${ }^{9}$, Q. Piel ${ }^{7}$, S. Pita ${ }^{25}$, V. Poireau ${ }^{7}$,

A. Priyana Noel ${ }^{13}$, D. A. Prokhorov ${ }^{18}$, H. Prokoph ${ }^{12}$, G. Pühlhofer ${ }^{15}$, M. Punch ${ }^{25,14}$, A. Quirrenbach ${ }^{30}$, S. Raab $^{19}$, R. Rauth $^{32}$,

P. Reichherzer ${ }^{9}$, A. Reimer ${ }^{32}$, O. Reimer ${ }^{32}$, Q. Remy ${ }^{4}$, M. Renaud ${ }^{29}$, F. Rieger ${ }^{4}$, L. Rinchiuso ${ }^{9}$, C. Romoli ${ }^{4}$, G. Rowell ${ }^{23}$, B. Rudak ${ }^{27}$,

E. Ruiz-Velasco ${ }^{4}$, V. Sahakian ${ }^{37}$, S. Sailer ${ }^{4}$, D. A. Sanchez ${ }^{7}$, A. Santangelo ${ }^{15}$, M. Sasaki ${ }^{19}$, M. Scalici ${ }^{15}$, F. Schüssler ${ }^{9}$,

H. M. Schutte ${ }^{1}$, U. Schwanke ${ }^{31}$, S. Schwemmer ${ }^{30}$, M. Seglar-Arroyo ${ }^{9}$, M. Senniappan ${ }^{14}$, A. S. Seyffert ${ }^{1}$, N. Shafi $^{21}$,

K. Shiningayamwe ${ }^{10}$, R. Simoni ${ }^{18}$, A. Sinha ${ }^{25}$, H. Sol ${ }^{16}$, A. Specovius ${ }^{19}$, S. Spencer ${ }^{8}$, M. Spir-Jacob ${ }^{25}$, . . Stawarz $^{13}$, L. Sun ${ }^{18}$,

R. Steenkamp ${ }^{10}$, C. Stegmann ${ }^{28,12}$, S. Steinmassl ${ }^{4}$, C. Steppa ${ }^{28}$, T. Takahashi ${ }^{38,39}$, T. Tavernier ${ }^{9}$, A. M. Taylor ${ }^{12}$, R. Terrier ${ }^{25}$,

D. Tiziani ${ }^{19}$, M. Tluczykont ${ }^{24}$, L. Tomankova ${ }^{19}$, C. Trichard $^{2}$, M. Tsirou ${ }^{29}$, R. Tuffs ${ }^{4}$, Y. Uchiyama ${ }^{33}$, D. J. van der Walt ${ }^{1}$,

C. van Eldik $^{19}$, C. van Rensburg ${ }^{1}$, B. van Soelen ${ }^{35}$, G. Vasileiadis ${ }^{29}$, J. Veh ${ }^{19}$, C. Venter ${ }^{1}$, P. Vincent ${ }^{17}$, J. Vink ${ }^{18}$, H. J. Völk ${ }^{4}$,

T. Vuillaume ${ }^{7}$, Z. Wadiasingh ${ }^{1}$, S. J. Wagner ${ }^{30}$, J. Watson ${ }^{8}$, F. Werner ${ }^{4}$, R. White ${ }^{4}$, A. Wierzcholska ${ }^{11,30}$, Yu Wun Wong ${ }^{19}$,

A. Yusafzai ${ }^{19}$, M. Zacharias ${ }^{1,16}$, R. Zanin ${ }^{4}$, D. Zargaryan ${ }^{3,5}$, A. A. Zdziarski ${ }^{27}$, A. Zech ${ }^{16}$, S. J. Zhu ${ }^{12}$, A. Ziegler ${ }^{19, \star}$,

J. Zorn ${ }^{4}$, S. Zouari ${ }^{25}$, and N. Żywucka ${ }^{1}$

(Affiliations can be found after the references)

Received 6 July 2020 / Accepted 27 September 2020

\begin{abstract}
The unidentified very-high-energy (VHE; $E>0.1 \mathrm{TeV}$ ) $\gamma$-ray source, HESS J1826-130, was discovered with the High Energy Stereoscopic System (HESS) in the Galactic plane. The analysis of $215 \mathrm{~h}$ of HESS data has revealed a steady $\gamma$-ray flux from HESS J1826-130, which appears extended with a half-width of $0.21^{\circ} \pm 0.02_{\text {stat }}^{\circ} \pm 0.05_{\text {sys }}^{\circ}$. The source spectrum is best fit with either a power-law function with a spectral index $\Gamma=1.78 \pm 0.10_{\text {stat }} \pm 0.20_{\text {sys }}$ and an exponential cut-off at $15.2_{-3.2}^{+5.5} \mathrm{TeV}$, or a broken power-law with $\Gamma_{1}=$ $1.96 \pm 0.06_{\text {stat }} \pm 0.20_{\text {sys }}, \Gamma_{2}=3.59 \pm 0.69_{\text {stat }} \pm 0.20_{\text {sys }}$ for energies below and above $E_{\text {br }}=11.2 \pm 2.7 \mathrm{TeV}$, respectively. The VHE flux from HESS J1826-130 is contaminated by the extended emission of the bright, nearby pulsar wind nebula, HESS J1825-137, particularly at the low end of the energy spectrum. Leptonic scenarios for the origin of HESS J1826-130 VHE emission related to PSR J1826-1256 are confronted by our spectral and morphological analysis. In a hadronic framework, taking into account the properties of dense gas regions surrounding HESS J1826-130, the source spectrum would imply an astrophysical object capable of accelerating the parent particle population up to $\gtrsim 200 \mathrm{TeV}$. Our results are also discussed in a multiwavelength context, accounting for both the presence of nearby supernova remnants, molecular clouds, and counterparts detected in radio, X-rays, and TeV energies.
\end{abstract}

Key words. ISM: supernova remnants - ISM: clouds - gamma rays: general - gamma rays: ISM

^ Corresponding authors; e-mail: contact .hess@hess-experiment.eu 


\section{Introduction}

The High Energy Stereoscopic System (HESS) Galactic Plane Survey (HGPS) revealed the presence of 16 new $\gamma$-ray sources in the very high energy (VHE; $E>0.1 \mathrm{TeV}$ ) domain (H.E.S.S. Collaboration 2018). Among them, HESS J1826-130 is located in a region that is exceptionally rich in VHE $\gamma$-ray sources, also encompassing the nearby pulsar wind nebula (PWN) HESS J1825-137 and the $\gamma$-ray binary system, LS 5039. HESS J1826-130 belongs to a growing class of VHE $\gamma$-ray sources affected by source confusion, caused by the influence of luminous neighboring $\gamma$-ray emitters, similar to the case of HESS J1641-463 (Abramowski et al. 2014). HESS J1826-130 is located just $\sim 0.7^{\circ}$ to the north of HESS J1825-137 (H.E.S.S. Collaboration 2019), a bright PWN whose extended emission prevented an earlier discovery of HESS J1826-130 at VHEs.

While HESS J1826-130 was clearly distinguishable from HESS J1825-137 in the HGPS, a deep study accounting for its morphological and spectral properties beyond the results obtained with the standard HGPS analysis tools was still lacking. In this paper, the results obtained from the analysis of $215 \mathrm{~h}$ of VHE $\gamma$-ray data recorded with the HESS telescopes is presented, yielding an accurate morphological description and a detailed spectral characterization of the source, including an energy-dependent emission study of the region. The results reported here on HESS J1826-130 are discussed in a multiwavelength context, discussing in particular its possible association with the spatially coincident supernova remnant, G18.45-0.42, and the Eel Nebula (Roberts et al. 2007), as well as with the VHE source 2HWC J1825-134 (Abeysekara et al. 2017), which displays emission up to energies of around $100 \mathrm{TeV}$.

Furthermore, HESS J1826-130 is surrounded by dense molecular clouds that provide support for a hadronic emission scenario. A recent investigation of the diffuse VHE emission around the Galactic center (GC) region demonstrated the presence of PeV particles within the central $10 \mathrm{pc}$ of the Galaxy (H.E.S.S. Collaboration 2016). This is the first robust detection of a VHE cosmic-ray (CR) accelerator which operates as a PeVatron. The MAGIC Collaboration recently published (MAGIC Collaboration 2020) the results of observations of the diffuse $\gamma$-ray emission in the vicinity of the GC. However, the GC alone would not be able to sustain the $\mathrm{CR}$ population close to $\mathrm{PeV}$ energies today unless the GC PeVatron was more powerful in the past. This opens up the possibility of other active $\mathrm{PeV}$ accelerators in the Galaxy that contribute to the observed Galactic CR flux around the spectral feature known as the knee, that is, a potentially new CR source population in the Galaxy. HESS J1826-130 may belong to such a new Galactic CR source population.

In Sect. 2, we present a summary of the multiwavelength (MWL) information available in the field of view (FoV) around HESS J1826-130. The HESS observations and data analysis are reported in Sect. 3, including morphological and spectral results, as well as a study of the contamination from the nearby PWN HESS J1825-137. Results are discussed in Sect. 4, with concluding remarks in Sect. 5.

\section{Multiwavelength environment}

As mentioned before, HESS J1826-130 is located in a region with several VHE $\gamma$-ray sources. At VHEs, the TeV sky around the source is dominated by one of the brightest and largest PWNs detected at VHEs, HESS J1825-137. This latter source is thought to be powered by PSR J1826-1334 (also known as PSR B1823-13), a powerful pulsar with a spin-down luminosity of $\sim 3 \times 10^{36} \mathrm{erg} \mathrm{s}^{-1}$, located about $13^{\prime}$ from the peak position of HESS J1825-137 in the VHE band. At GeV energies, both the spectral and the morphological properties of HESS J1825-137 resemble and smoothly connect with those observed at VHEs, as reported in Grondin et al. (2011) using the Fermi-LAT.

The first evidence for $\gamma$-ray emission originating from the north of PSR J1826-1334 was found by the detection of 3EG J1826-1302 (also called GeV J1825-1310) in 1999 with the EGRET telescope on-board the Compton Gamma Ray Observatory (Hartman et al. 1999). Follow-up X-ray observations of the same region led to the detection of a diffuse X-ray source, AX J1826.1-1300, in the 2-10 keV energy band (Roberts et al. 2001a). This X-ray source is characterized by two emission peaks located on top of a diffuse X-ray nebula. Further observations of AXJ1826.1-1300 in 2007 with the Chandra X-ray Observatory resolved the source in more detail (Roberts et al. 2007). The southern peak was found to be likely associated with a stellar cluster, whereas the northern peak was resolved and associated with a PWN. Due to a $4^{\prime}$ trail of hard X-ray emission connected to this new nebula, the authors called it the Eel Nebula. Roberts et al. (2007) were the first to introduce a new separate VHE $\gamma$-ray source north of HESS J1825-137, which they named HESS J1826-131 and associated with the Eel Nebula. Until that time, the remaining VHE emission to the north of HESS J1825-137 was thought to be connected to the bright source itself.

The Fermi-LAT detected the radio-quiet $\gamma$-ray pulsar PSR J1826-1256 (Abdo et al. 2009), with a spin down luminosity $\dot{\mathrm{E}}=3.6 \times 10^{36} \mathrm{erg} \mathrm{s}^{-1}$ and a characteristic age $\tau_{\mathrm{c}}=14.4 \mathrm{kyr}$, whose position is consistent with the Eel Nebula, representing a significant step forward in our understanding of the various sources contributing to the $\gamma$-ray emission from this region. In 2017, HESS J1826-130 was reported as a new source within the HGPS. The HGPS used a dedicated source-identification algorithm based on a multi-Gaussian component fit to a given region of interest covered by the survey (H.E.S.S. Collaboration 2018). In particular for HESS J1826-130, the spatially extended VHE $\gamma$-ray emission region matched the position of 3EG J1826-1302, also overlapping with the diffuse X-ray nebula observed with ASCA (which displays a radius of about 15'; see Fig. 11 in Roberts et al. 2001b) as well as the Eel Nebula (with an extension of about 4', Roberts et al. 2007). The latest results at VHEs of the region include the significant detection of an emission region, 2HWC J1825-134, included in the 2nd HAWC catalog (Abeysekara et al. 2017). Due to the large spatial extension of the HAWC emission region and the relatively low angular separation between HESS J1826-130 and HESS J1825-137, the two sources were not distinguishable in this catalog. Further studies, however, using a much larger dataset and employing updated analysis methods, provide a statistical improvement at the level of $\sim 5 \sigma$ when accounting for the presence of the two HESS VHE sources.

Within the surroundings of HESS J1826-130, there are also three supernova remnants (SNRs): G18.1-0.1, G18.6-0.2, and G18.45-0.42, which are detected in the radio band (Brogan et al. 2006; Anderson et al. 2017). Detailed studies of the gas dynamics and densities in the direction of the region north of HESS J1825-137 were carried out by Voisin et al. (2016), following the discovery of a molecular cloud north of PSR J1826-1334 by Lemiere et al. (2005). These studies revealed the presence of dense molecular clouds with particle density values up to $n_{\mathrm{H}_{2}} \sim 7 \times 10^{2} \mathrm{~cm}^{-3}$. The bulk of molecular gas in the vicinity of 


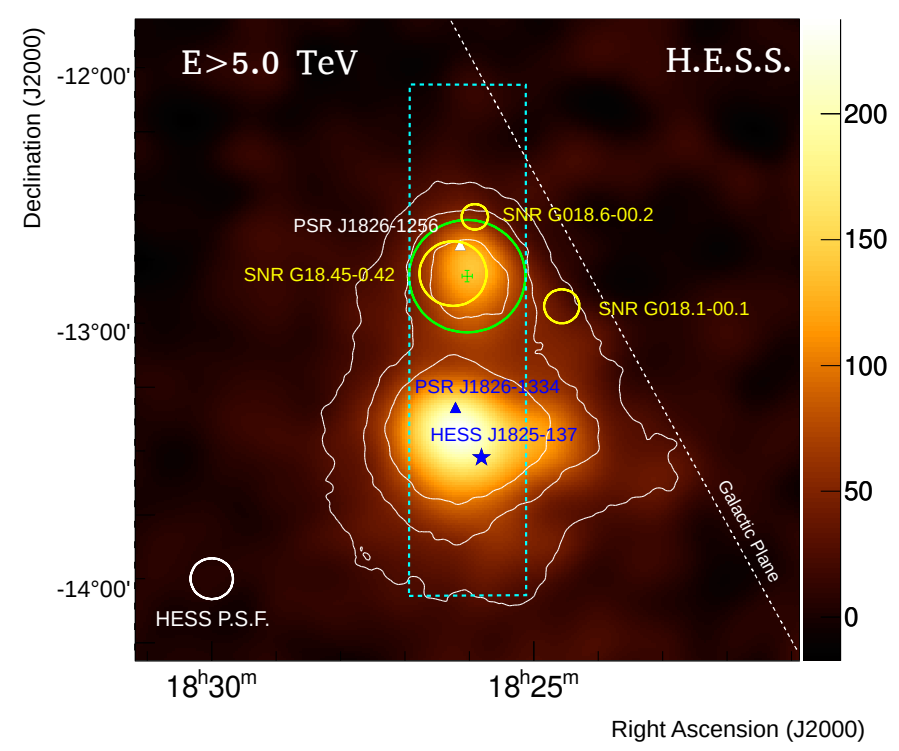

Fig. 1. Map of excess counts with energies $E>5 \mathrm{TeV}$ for region around HESS J1826-130 smoothed with a Gaussian kernel of $0.07^{\circ}$ to match HESS PSF at the same energies. The $68 \%$ containment radius of the HESS PSF is shown with the white circle in the lower-left corner. The white contours indicate the significance of the emission at the $5 \sigma, 10 \sigma$, and $15 \sigma$ level. The color scale is in units of excess counts per smoothing Gaussian width. The green circle shows the integration region used for deriving the source spectrum (Fig. 3), while the green cross indicates the value and $1 \sigma$ uncertainty of the best-fit position of the source. The nearby SNRs: G18.45-0.42, G018.6-00.2, and G018.1-00.1 are represented with yellow circles, while the white triangle indicates the position of the $\gamma$-ray pulsar PSR J1826-1256. The blue star shows the peak position of the bright nearby source HESS J1825-137 for energies above $0.25 \mathrm{TeV}$ (we employed the position as reported in H.E.S.S. Collaboration 2019 since the data set analyzed here accounts for a total exposure across all of HESS J1825-137, which is less than that used in its dedicated study). The blue triangle indicates the position of PSR J1826-1334. The white dashed line indicates the orientation and position of the Galactic plane, while the cyan dashed rectangle indicates the area for the extraction of the profiles shown in Fig. 2.

HESS J1826-130 is located at $V_{\mathrm{LSR}}=45-60 \mathrm{~km} \mathrm{~s}^{-1}$, corresponding to a kinematic distance of $4.0 \mathrm{kpc}$, and $V_{\mathrm{LSR}}=60-80 \mathrm{~km} \mathrm{~s}^{-1}$, corresponding to a kinematic distance of $4.6 \mathrm{kpc}$. The former gas distance estimate is consistent with the dispersion measurement of PSR J1826-1334. Voisin et al. (2016) concluded that due to the general spatial match between the molecular gas and the $\mathrm{TeV}$ emission, HESS J1826-130 might be explained by a hadronic emission scenario, with the progenitor SNR of HESS J1825-137 being a suitable local CR source candidate. However, a leptonic scenario connected to the Eel Nebula could not be ruled out. Furthermore, the two SNRs, G18.1-0.1 and G18.6-0.2, were considered unlikely to be directly related to the $\mathrm{TeV}$ excess due to their offset positions and their small angular diameters.

\section{HESS observations and data analysis results}

The High Energy Stereoscopic System is an array of five imaging atmospheric Cherenkov telescopes located in the Khomas Highland of Namibia, $1800 \mathrm{~m}$ above sea level. HESS in phase I comprised four $12 \mathrm{~m}$ diameter telescopes that have been fully operational since 2004. A fifth telescope (CT5), with a larger mirror diameter of $28 \mathrm{~m}$ and newly designed camera (Bolmont et al. 2014), was added to the center of the array and has been

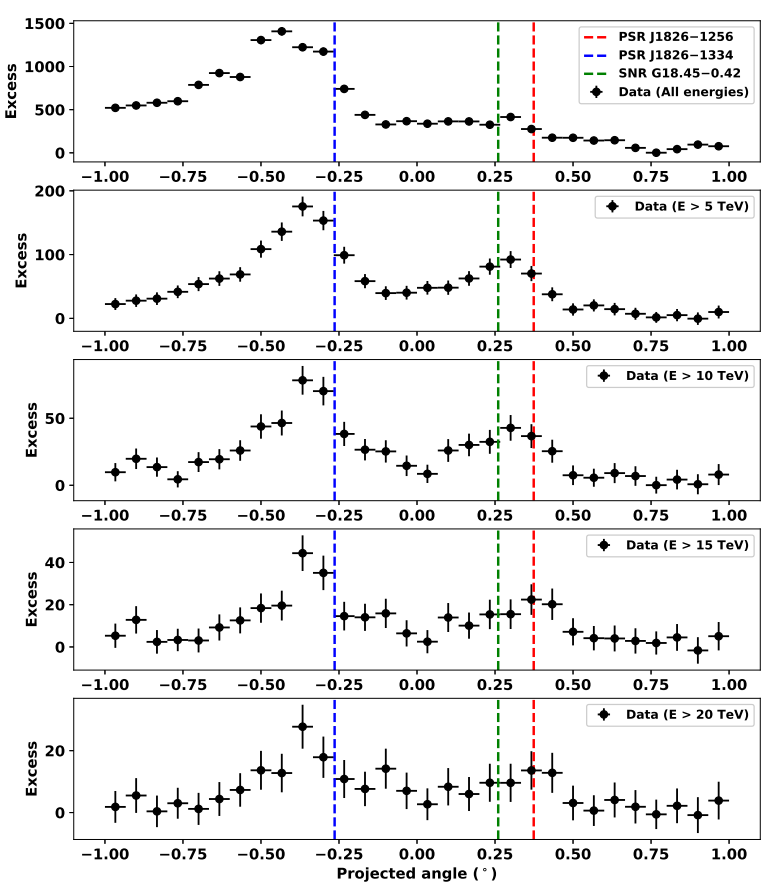

Fig. 2. Distribution of VHE $\gamma$-ray excess along axis of the cyan dashed rectangle indicated in Fig. 1 that encompasses both HESS J1825-137 (broad peak on the left) and HESS J1826-130 (shallower peak on the right, becoming apparent only at high energies) above $0.4 \mathrm{TeV}$ and at $E>5,10,15$, and $20 \mathrm{TeV}$. Vertical lines show the position of PSR J1826-1256 (red), PSR J1826-1334 (blue) and SNR G018.450.42 (red).

operational since September 2012. The HESS phase I array configuration is sensitive to $\gamma$-ray energies between $100 \mathrm{GeV}$ and several tens of $\mathrm{TeV}$, while the energy threshold of the array was lowered to 10's of GeV with the addition of CT5. The VHE HESS data presented in this paper were recorded with the HESS phase I array configuration, which can measure extensive air showers with an angular resolution better than $0.1^{\circ}$ and an average energy resolution of $15 \%$ for an energy of $1 \mathrm{TeV}$ (Aharonian et al. 2006a).

The VHE $\gamma$-ray observations of the FoV around HESS J1826-130 were carried out between 2004 and 2015. About $140 \mathrm{~h}$ of observations were recorded with the HESS phase I array configuration (between 2004 and 2012), while $\sim 95 \mathrm{~h}$ were obtained with the HESS phase II array configuration in a split observation mode without participation of the CT5 telescope. These data sets provide a total acceptance-corrected live-time of $215 \mathrm{~h}$ of HESS data after the application of quality selection criteria (Aharonian et al. 2006a).

The data have been analyzed with the HESS Analysis Package (HAP) for shower reconstruction, and a multivariate analysis technique (Ohm et al. 2009) was applied to provide an improved distinction between hadron and $\gamma$-ray events. A crosscheck analysis was performed using an independent calibration and analysis method (de Naurois \& Rolland 2009), which gives compatible results with the main analysis.

\subsection{Detection and morphological analysis}

In order to achieve an improved angular resolution and reduce strong contamination coming from the nearby bright source, HESS J1825-137, the source position and morphology of HESS J1826-130 were obtained with a cut configuration 
Table 1. Analysis statistics of the HESS J1826-130 region using different energy thresholds $\left(E_{\mathrm{th}}\right)$ corresponding to the VHE $\gamma$-ray excess profiles shown in Fig. 2.

\begin{tabular}{cccccc}
\hline \hline$E_{\text {th }}(\mathrm{TeV})$ & $N_{\text {On }}$ & $N_{\text {Off }}$ & $\alpha$ & Excess & Significance $(\sigma)$ \\
\hline 0.4 & 5544 & 27006 & 0.145 & 1627.6 & 22.6 \\
5 & 569 & 1685 & 0.132 & 346.2 & 17.8 \\
10 & 237 & 664 & 0.126 & 153.4 & 12.6 \\
15 & 118 & 363 & 0.125 & 72.5 & 8.2 \\
20 & 68 & 220 & 0.120 & 41.6 & 6.2 \\
\hline
\end{tabular}

Notes. The events below the energy threshold shown in the $E_{\text {th }}$ column were not taken into account in the analysis. The number of events within the source region $\left(N_{\text {On }}\right)$, within the ring region used for estimating the background level $\left(N_{\text {Off }}\right)$, the alpha factor $(\alpha)$, the corresponding number of excess events, and the Li\&Ma statistical significance (Li \& Ma 1983) are given for each energy threshold separately.

optimized for high energies that requires a minimum of 160 photo-electrons per image. The cosmic-ray background level was estimated using the ring background model (Berge et al. 2007). Using the full $215 \mathrm{~h}$ dataset, HESS J1826-130 was detected with a statistical significance of $22.6 \sigma^{1}$, determined following Eq. (17) in Li \& Ma (1983). Figure 1 shows the acceptancecorrected VHE $\gamma$-ray excess map of the region around HESS J1826-130 at energies greater than $5 \mathrm{TeV}$, smoothed with the HESS point spread function (PSF) with a radius of $0.07^{\circ}$.

Until 2017, in the first two publications by the H.E.S.S. Collaboration concerning HESS J1825-137 (Aharonian et al. 2006b,c), HESS J1826-130 remained unnoticed and hidden to the standard source detection techniques due to its low brightness with respect to HESS J1825-137. Confirmed as a separate source in 2017 (H.E.S.S. Collaboration 2018), HESS J1826-130 emerges toward higher energies as illustrated in Fig. 2 and corresponding analysis statistics given in Table 1, with reduced contamination from HESS J1825-137, potentially due to its energy-dependent morphology and hinting at a harder VHE spectrum.

The best-fit position and extension of the source have been determined by convolving 2D Gaussian models with the HESS PSF and fitting this to the excess event distribution (for $E>$ $0.4 \mathrm{TeV}$ ). The HESS PSF was derived from simulations and modeled as a weighted sum of three 2D Gaussian functions for the morphology analysis. A log-likelihood ratio test (LLRT) was used to select the best morphology model that represents the HESS data. The centroid of the 2D Gaussian corresponding to the best-fit position of HESS J1826-130 is RA (J2000): $18^{\mathrm{h}} 26^{\mathrm{m}} 02.16^{\mathrm{s}} \pm 3.38_{\text {stat }}^{\mathrm{s}} \pm 1.30_{\text {sys }}^{\mathrm{s}}$ and Dec $(\mathrm{J} 2000):-13^{\circ} 04^{\prime} \pm$ $1.0^{\prime}{ }_{\text {stat }} \pm 0.3^{\prime}$ sys. The extension (radius) of the source is estimated to be $0.21^{\circ} \pm 0.02_{\text {stat }}^{\circ} \pm 0.05_{\text {sys }}^{\circ}$, which is significantly larger than the HESS PSF.

\subsection{Spectral analysis}

The green circular region with a radius of $0.22^{\circ}$ centered at the best-fit position of HESS J1826-130 (see Fig. 1) was used as the integration region for measuring the differential VHE $\gamma$-ray spectrum of the source. The reflected background model (Berge et al. 2007) was used to estimate the background. The source spectrum was derived using the forward folding technique (Piron

1 For comparison, HESS J1826-130 is detected at a statistical significance of about $9.4 \sigma$ in the HGPS (H.E.S.S. Collaboration 2018).

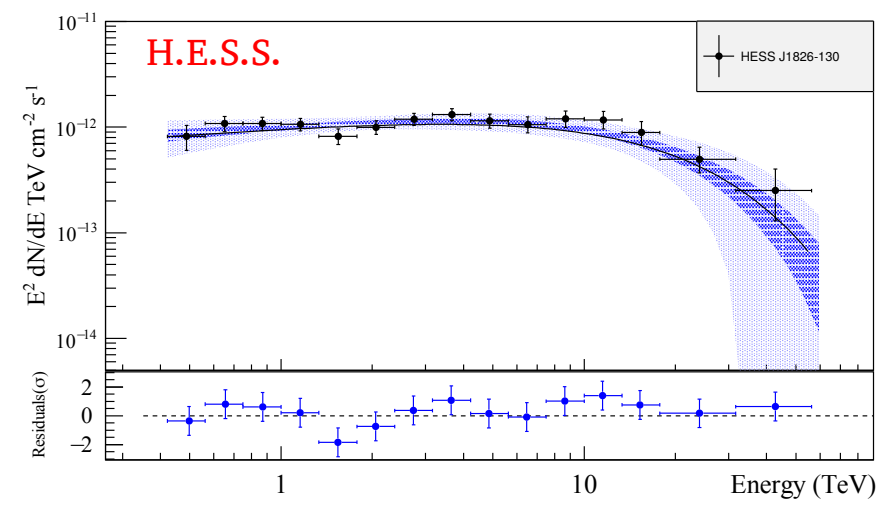

Fig. 3. VHE $\gamma$-ray spectrum of HESS J1826-130 extracted from the green circular source region shown in Fig. 1. The black dots show the flux points with $1 \sigma$ statistical errors. The $1 \sigma$ and $3 \sigma$ error bands of the best-fit ECPL model are shown with the blue and light blue shaded regions, respectively, while the best-fit ECPL model itself is shown with the black line. The spectrum shown is binned such that all flux points have a minimum significance level of $2.0 \sigma$. The significance of the last flux bin centered at $42.7 \mathrm{TeV}$ is $2.4 \sigma$.

et al. 2001). Several spectral models were used to fit the data, including a simple power-law (PL) model, a broken power-law (BPL) model, and a power-law model with an exponential cutoff (ECPL; see Table 2). The spectral fit is performed between 0.42 and $56.2 \mathrm{TeV}$. The best-fit model is found to be the ECPL, with a $p$-value $=0.41$ and a likelihood ratio test (LLRT) against the PL model providing a statistical improvement at the $\sim 3.2 \sigma$ level. The differential VHE emission is thus best represented by $\mathrm{d} N / \mathrm{d} E=\Phi_{0}(E / 1 \mathrm{TeV})^{-\Gamma} \exp \left(-E / E_{\mathrm{c}}\right)$, with $\Phi_{0}=(10.10 \pm$ $\left.0.69_{\text {stat }} \pm 2.02_{\text {sys }}\right) \times 10^{-13} \mathrm{~cm}^{-2} \mathrm{~s}^{-1} \mathrm{TeV}^{-1}, \Gamma=1.78 \pm 0.10_{\text {stat }} \pm$ $0.20_{\text {sys }}$ and a cut-off energy of $E_{\mathrm{c}}=15.2^{+5.5} \mathrm{TeV}$ as shown in Fig. 3. The integral flux level above $1 \mathrm{TeV}^{2}$ is $\Phi(>1 \mathrm{TeV})=$ $\left(10.2 \pm 0.5_{\text {stat }} \pm 2.0_{\text {sys }}\right) \times 10^{-13} \mathrm{~cm}^{-2} \mathrm{~s}^{-1}$ and corresponds to $4.9 \%$ of the Crab Nebula flux at the same energies ${ }^{2}$. A $2 \sigma$ lower limit on the cut-off energy of the source is derived at $\sim 10 \mathrm{TeV}$. A fit to a BPL model gives a break energy at $11.2 \pm 2.7 \mathrm{TeV}$, which is compatible with the cut-off energy obtained from the ECPL model. The comparison between the ECPL and the BPL model does not show any preference from obtained p-values. Both models clearly indicate a spectral steepening of the spectrum above $10 \mathrm{TeV}$. The integral flux is found to be constant within the HESS dataset at different timescales, from run-wise ( $\sim 30 \mathrm{~min})$ to year-wise light curves.

The integration region used to measure the VHE spectrum of HESS J1826-130 is strongly contaminated, especially at lower energies, by the relatively softer spectrum of HESS J1825-137. Therefore, the obtained spectrum is affected by this contamination. Taking into account the relatively softer contribution from the contaminating source, the intrinsic spectrum of HESS J1826-130 is expected to be even harder with respect to the spectral results discussed above.

\subsection{Contamination study}

The estimation of intrinsic spectra for sources strongly contaminated by neighboring bright and extended sources presents a nontrivial challenge. Although morphology studies in different energy bands can provide a powerful tool for new discoveries,

2 Throughout this paper, $1 \mathrm{Crab}$ unit is defined here as $\Phi(>1 \mathrm{TeV})=$ $2.26 \times 10^{-11} \mathrm{~cm}^{-2} \mathrm{~s}^{-1}$ (Aharonian et al. 2006a). 
H.E.S.S. Collaboration: An extreme particle accelerator in the Galactic plane: HESS J1826-130

Table 2. Comparison of different spectral models fit to the data in the energy range of $[0.4,56.2] \mathrm{TeV}$, together with the spectral parameter values.

\begin{tabular}{cccccc}
\hline \hline Spectral model & $\begin{array}{c}\text { Flux }(1 \mathrm{TeV}) \\
\times 10^{-13} \mathrm{~cm}^{-2} \mathrm{~s}^{-1} \mathrm{TeV}^{-1}\end{array}$ & $\begin{array}{c}\text { Int. flux }(>1 \mathrm{TeV}) \\
\times 10^{-13} \mathrm{~cm}^{-2} \mathrm{~s}^{-1}\end{array}$ & Parameters & $\begin{array}{c}-2 * \text { Loglike } \\
(\text { LLRT })\end{array}$ & $\begin{array}{c}\chi^{2} / n . d . f \\
(p \text {-val })\end{array}$ \\
\hline Power law & $11.1 \pm 0.6$ & $\begin{array}{c}9.8 \pm 0.4 \\
(4.7 \% \mathrm{Crab})\end{array}$ & $\Gamma=2.12 \pm 0.04$ & 44.015 & $37.3 / 10$ \\
& & $10.2 \pm 0.5$ & $\Gamma=1.78 \pm 0.10$ & 33.566 & $9.3 / 9$ \\
& $10.1 \pm 0.7$ & $(4.9 \% \mathrm{Crab})$ & $\lambda=0.0659 \pm 0.0177\left(\mathrm{TeV}^{-1}\right)$ & $(3.2 \sigma)$ & $(0.41)$ \\
\hline $\begin{array}{c}\text { Exp. cut-off } \\
\text { power law }\end{array}$ & $10.1 \pm 0.5$ & $\Gamma_{1}=1.96 \pm 0.06$ & 32.577 & $8.6 / 8$ \\
\hline Broken & $10.2 \pm 0.7$ & $(4.8 \% \mathrm{Crab})$ & $\Gamma_{2}=3.59 \pm 0.69$ & $(\mathrm{n} / \mathrm{a})$ & $(0.38)$ \\
power law & & & $\mathrm{E}_{\mathrm{br}}=11.2 \pm 2.7(\mathrm{TeV})$ & & \\
\hline
\end{tabular}

Notes. In all cases, statistical errors are quoted, while systematics errors are $20 \%$ for flux and 0.2 for spectral indices. The likelihoods and $p$-values are given for each model for comparison.

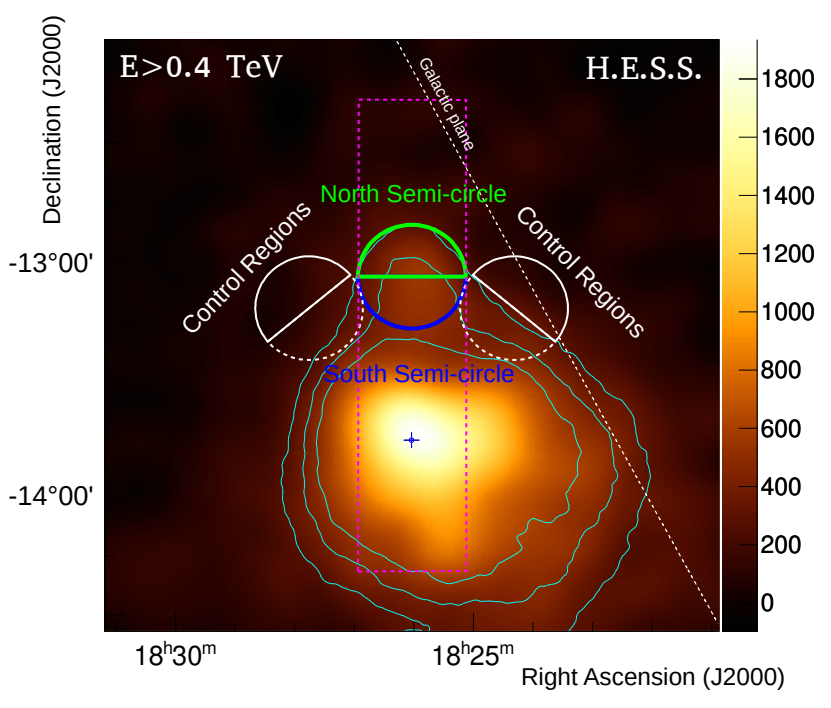

Fig. 4. VHE $\gamma$-ray excess map of HESS J1826-130 for all events saturated for a better visualization, showing the north semi-circle (green), south semi-circle (blue), and control regions for both north (solid white) and south (dashed white) semi-circle regions. The control regions are distributed in a radially symmetric manner around the peak position of HESS J1825-137, indicated with the blue cross, obtained from the dataset and for energies above $0.4 \mathrm{TeV}$. The cyan contours indicate the significance of the emission at the $15 \sigma, 20 \sigma$ and $25 \sigma$ levels.

such a method does not help in providing a quantitative characterization of the contamination, as systematic uncertainties in the flux estimation are expected to dominate. In general, contamination effects can be neglected if they are at the $10 \%$ level or lower, since this is, for example, the typical HESS systematic error on the source flux. For contamination exceeding 10\%, in particular for spectral studies, a dedicated analysis is needed.

Performing contamination studies for HESS J1826-130 requires knowledge about the energy-dependent morphology of HESS J1825-137, which is highly asymmetrical. The contamination was estimated in this case using the morphology model that represents the HESS data best fit. The model includes a 2D Gaussian function accounting for HESS J1826-130 along with other 2D Gaussian functions representing the other sources, HESS J1825-137 and the gamma-ray binary system LS 5039, present in the FoV. Taking the ratio of the total number of excess counts within the integration region (Fig. 1, green circle) used for measuring HESS J1826-130's spectrum between the model components can give a quantitative estimation of the contamination. By using such an approach, the contamination from HESS J1825-137 is estimated to be $\sim 40 \%$ below $1.5 \mathrm{TeV}$ and $\sim 20 \%$ above $1.5 \mathrm{TeV}$, while it is strongly reduced $(<10 \%)$ at energies above $2.0 \mathrm{TeV}$. Given the relatively large angular distance of LS 5039 to the integration region (around $2.0^{\circ}$ ) encompassing HESS J1826-130, we find that the contamination from the binary system is negligible.

The spectral contamination from HESS J1825-137 has been estimated based on the fact that as the separation between contaminated and contaminating source increases, one expects the effect of contamination to decrease. To test the biasing impact of the contamination, the HESS J1826-130 integration region was split into a near and far half-circle, referred to as the south and north semi-circles shown in Fig. 4. Spectra from two control regions at both sides of the source position were also extracted in order to determine whether or not the contribution from HESS J1825-137 for the north (south) semi-circle is significant. The spectra of the upper halves of these control regions (solid semi-circles in Fig. 4) show insignificant $(<5.0 \sigma)$ emission, while the lower halves of the control regions (dashed semi-circles in Fig. 4), which are closer to HESS J1825-137, show significant $(>5.0 \sigma)$ VHE $\gamma$-ray emission.

One expects that the contamination in the north semi-circle region should be less with respect to the south semi-circle region, and the spectrum should reflect the intrinsic spectral properties of HESS J1826-130. The spectral fit results, assuming an ECPL model and obtained from the north and south semi-circle regions, are given in Table 3 along with the total results. As expected, the north semi-circle spectrum is slightly harder than those of the total and south semi-circle regions. Although the contamination from HESS J1825-137 could potentially affect the spectrum of HESS J1826-130, our studies demonstrate that the effects on the spectral parameters are at the level of our analysis systematic uncertainties. The intrinsic spectrum of HESS J1826-130, corrected for this contamination, is found to be well described by an ECPL model where $\Phi_{0}=9.2 \times$ $10^{-13} \mathrm{~cm}^{-2} \mathrm{~s}^{-1} \mathrm{TeV}^{-1}, \Gamma=1.7$ and a cut-off energy around $E_{\mathrm{c}}=$ $16 \mathrm{TeV}$.

A further investigation of the intrinsic spectrum of HESS J1826-130 was performed using a 3D cube-analysis approach $^{3}$. With such a template-based analysis method, uncontaminated spectra can be derived, since all sources located

\footnotetext{
3 We used ctools (Knödlseder et al. 2016) and gammapy (Deil et al. 2017), which include 3D cube analysis approaches optimized for measuring intrinsic spectra from contaminated sources.
} 
Table 3. Comparison of ECPL spectral models for the HESS J1826-130 integration region (Total), plus the north and south semi-circle regions.

\begin{tabular}{ccccc}
\hline \hline Region & $\begin{array}{c}\text { Normalization } \\
\left(10^{-13} \mathrm{~cm}^{-2} \mathrm{~s}^{-1} \mathrm{TeV}^{-1}\right)\end{array}$ & Index & $\begin{array}{c}\text { Cut-off energy } \\
(\mathrm{TeV})\end{array}$ & $\begin{array}{c}\text { Integral flux }(>1 \mathrm{TeV}) \\
(\% \text { Crab Nebula })\end{array}$ \\
\hline Total & $10.10 \pm 0.69_{\text {stat }} \pm 2.02_{\text {sys }}$ & $1.78 \pm 0.10_{\text {stat }} \pm 0.20_{\text {sys }}$ & $15.2_{-3.2}^{+5.5}$ & 4.9 \\
North semi-circle & $4.64 \pm 0.49_{\text {stat }} \pm 0.93_{\text {sys }}$ & $1.69 \pm 0.14_{\text {stat }} \pm 0.20_{\text {sys }}$ & $15.8_{-4.3}^{+9.6}$ & 2.4 \\
South semi-circle & $5.06 \pm 0.49_{\text {stat }} \pm 1.01_{\text {sys }}$ & $1.88 \pm 0.14_{\text {stat }} \pm 0.20_{\text {sys }}$ & $16.4_{-4.7}^{+11.9}$ & 2.3 \\
\hline
\end{tabular}

Notes. The integral flux (in \% Crab Nebula) is given for the same energy range (above $1 \mathrm{TeV}$ ).

in the FoV are simultaneously taken into account. The 3D analysis results published in Ziegler (2018) are found to be compatible with the results presented here.

\section{Discussion}

The study of VHE $\gamma$-ray sources displaying spectra extending above several tens of $\mathrm{TeV}$ is required to understand the origin of the highest energy CRs close to the knee in the CR spectrum. These studies are particularly promising in cases in which dense gas regions are coincident with the source of interest. In the case of HESS J1826-130, the spatial coincidence of a dense molecular hydrogen region found along the line of sight (see Voisin et al. 2016), suggests that the radiation could actually be produced by protons with energies of several hundred TeV colliding with this gas. Figure 5 displays the HESS excess map obtained at energies above $20 \mathrm{TeV}$ together with column density profiles derived from Nobeyama ${ }^{12} \mathrm{CO}(1-0)$ (Umemoto et al. 2017) and SGPS HI data (McClure-Griffiths et al. 2005), for two different gas velocity ranges, $45-60 \mathrm{~km} \mathrm{~s}^{-1}$ (corresponding to a kinematic distance of $4.0 \mathrm{kpc}$ ) and $60-80 \mathrm{~km} \mathrm{~s}^{-1}$ (corresponding to a kinematic distance of $4.6 \mathrm{kpc}$ ). The scaling $\mathrm{X}$-factors, $\mathrm{X}_{\mathrm{HI}}=1.8 \times 10^{18} \mathrm{~cm}^{-2} \quad\left(\mathrm{~K} \quad \mathrm{~km} \mathrm{~s}^{-1}\right)^{-1}$ and $\mathrm{X}_{\mathrm{CO}}=2.0 \times$ $10^{20} \mathrm{~cm}^{-2}\left(\mathrm{~K} \mathrm{~km} \mathrm{~s}^{-1}\right)^{-1}$, were taken from Dickey \& Lockman (1990) and Strong et al. (2004), respectively. The Nobeyama ${ }^{12} \mathrm{CO}(1-0)$ data was smoothed to match the SGPS beam size of 2'. The $\mathrm{CO}$ and $\mathrm{HI}$ distribution display two local maxima that do not coincide with the $E>20 \mathrm{TeV}$ peak, although they still provide target densities at the level of $\sim 5 \times 10^{2} \mathrm{~cm}^{-3}$ at the position of HESS J1826-130.

The best-fit parent proton population in this scenario would have a spectral index of $\sim 1.7$ and a cut-off energy around $200 \mathrm{TeV}^{4}$. The relative hardness of the photon spectrum could be due to the highest energy protons diffusing into dense clouds, while lower energy protons might still be confined within the accelerating source, or efficiently excluded from the clouds if the diffusion coefficient inside the clouds is suppressed (Gabici et al. 2009). A hadronic scenario in which runaway protons accelerated by one of the coincident or nearby SNRs emit TeV photons when interacting with the dense ambient gas found along the line of sight could explain the hard spectrum of HESS J1826-130. We note, however, that the filled centers of SNR G18.5-0.04 and SNR G18.1-00.1 are possibly too old to be able to accelerate protons up to hundreds of TeV. The $\gamma$-ray luminosity, $L_{\gamma}$, of the source is $8 \times 10^{33} \mathrm{erg} \mathrm{s}^{-1}$ for a distance of $4 \mathrm{kpc}$, corresponding to the estimated distances of the SNRs G18.45-0.42 (Karpova et al. 2019), G018.1-00.1, or G018.6-00.25 . This would translate

\footnotetext{
4 We employed the NAIMA fitting software package for these computations (Zabalza 2015).

5 We note that Voisin et al. (2016) discuss the possibility of a larger distance to G018.6-00.2 of $11.4 \mathrm{kpc}$, disfavoring an association of HESS J1826-130 with G018.6-00.2.
}

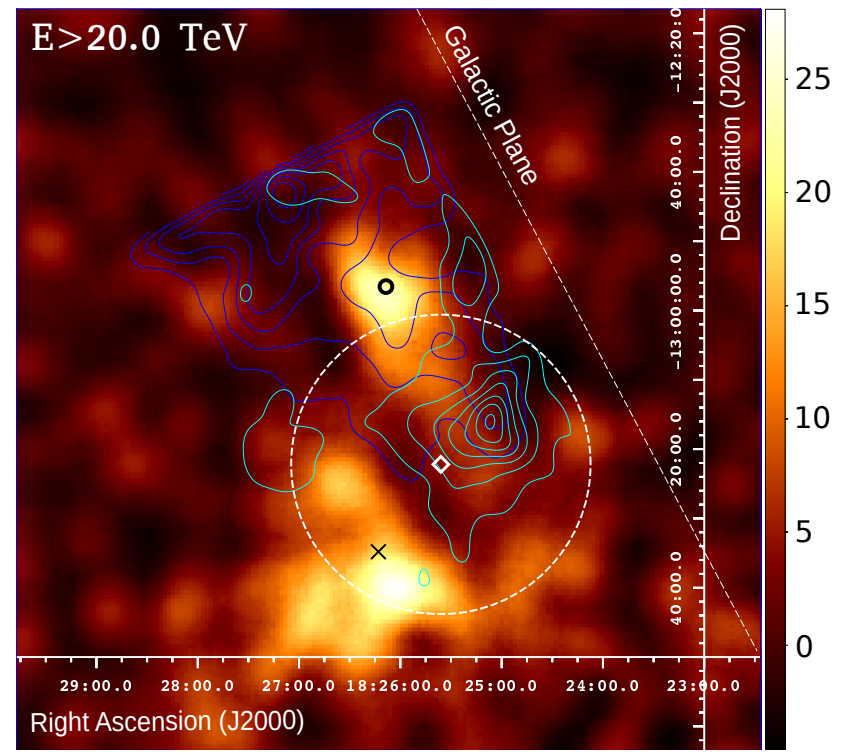

Fig. 5. VHE $\gamma$-ray excess map of HESS J1826-130 for events above $20 \mathrm{TeV}$, together with total column density map contours. The black circle and cross show the location of the pulsars PSR J1826-1256 and PSR J1826-1334. The cyan and blue contours show the total column density contours of $[2.5,9.5] \times 10^{22} \mathrm{~cm}^{2}$, derived from Nobeyama ${ }^{12} \mathrm{CO}(1-0)$ and SGPS HI data, for gas velocities between $45-60 \mathrm{~km} \mathrm{~s}^{-1}$ (d: $4 \mathrm{kpc}$ ) and 60-80 $\mathrm{km} \mathrm{s}^{-1}$ (d: $4.6 \mathrm{kpc}$ ), respectively. The white dashed circle and diamond are the extension and best-fit position of recently reported $E>100 \mathrm{TeV}$ emitting source, eHWC J1825-134 (HAWC Collaboration 2020).

into an energy output in accelerated protons $W_{\mathrm{pp}}=L_{\gamma} \times t_{\mathrm{pp}}$, of $6 \times 10^{49}\left(n / 1 \mathrm{~cm}^{-3}\right)^{-1} \mathrm{erg}$, where the gas density value is $n=$ $5 \times 10^{2} \mathrm{~cm}^{-3}$ and $t_{\mathrm{pp}}$ is the typical timescale for gamma-rays to be produced through neutral pion decay following the collision of relativistic protons.

The relatively hard spectrum found at VHE gamma rays could also be produced in a leptonic scenario by an electron population with a spectral index close to 2.0 and a cut-off at around $70 \mathrm{TeV}$. These electrons could be accelerated by the pulsar PSR J1826-1256 and up-scatter cosmic microwave background (CMB) and IR photons to produce the observed $\mathrm{TeV}$ emission. PSR J1826-1256 is powering the Eel Nebula (PWN G018.5-0.4, Roberts et al. 2007), which displays an elongated $\mathrm{X}$-ray trail of shocked material associated with its fast proper motion through the surrounding medium. An estimate for the magnetic field inside the Eel Nebula of $\sim 20-30 \mu \mathrm{G}$ is provided in Keogh (2010). Recently, Duvidovich et al. (2019) made use of XMM-Newton observations of the region around PSR J18261256 and discussed such a possibility. Based on the spectral softening found along the nebula, they considered X-rays produced by electrons with energies $E_{\mathrm{e}} \lesssim 150 \mathrm{TeV}$ radiating 
synchrotron emission in a magnetic field $B>2 \mu \mathrm{G}$. In a parallel study, Karpova et al. (2019) also reported on the X-ray analysis of this very same $X M M$-Newton dataset, complemented with additional $\sim 90 \mathrm{ks}$ of Chandra observations of the region. These data support the association of HESS J1826-130 with the Eel Nebula, although somewhat harder spectral indices close to the PWN are derived $\left(\Gamma_{\mathrm{X}} \sim 1.2\right)$, followed by softer emission, with $\Gamma_{\mathrm{X}} \sim 2.5$ at larger distances along the PWN tail.

The excess map reported in Fig. 5 at $E>20 \mathrm{TeV}$ traces emission from the vicinity of PSR J1826-1256. TeV photons could be produced by these synchrotron X-ray-emitting electrons through inverse Compton scattering off the CMB and IR photon fields. For the latter, an energy density at a distance of $4 \mathrm{kpc}$ $\sim 0.23 \mathrm{eV} \mathrm{cm}^{-3}$ was assumed (see e.g., Vernetto \& Lipari 2016; we note, however, that the distance to PSR J1826-1256 is not very well constrained; e.g., Wang 2011 favored a distance of about $1.2 \mathrm{kpc}$, whereas Karpova et al. 2019 found a distance of $\sim 3.5 \mathrm{kpc}$ based on an empirical interstellar absorptiondistance correlation). The CMB photon field provides $u_{\mathrm{CMB}} \sim 0.26 \mathrm{eV} \mathrm{cm}^{-3}$. Electron Lorentz factors of $\gamma_{\mathrm{e}} \sim 1.3 \times 10^{8}$ and $\gamma_{\mathrm{e}} \sim 3.6 \times 10^{7}$ would be required to reach the VHE $\gamma$-ray fluxes reported here for CMB and IR seed photons, respectively, in agreement with the estimates in Duvidovich et al. (2019).

The complex region around HESS J1826-130 has also been observed at $\mathrm{TeV}$ energies with the HAWC telescopes. A spatially coincident source, 2HWC J1825-13, is included in the 2nd HAWC catalog (Abeysekara et al. 2017). Furthermore, the HAWC collaboration recently reported the detection of $E>100 \mathrm{TeV}$ emission from eHWC J1825-134 (HAWC Collaboration 2020). Its position and extension are marked with a white dotted circle in Fig. 5. With a half-width of $\sim 0.36^{\circ}$, the source encompasses a local peak of the ${ }^{12} \mathrm{CO}$ distribution. It is also compatible with the location of the $E>20 \mathrm{TeV} \gamma$-rays from HESS J1825-137, and adjacent to HESS J1826-130 emission $E>20 \mathrm{TeV}$. On spectral grounds, both HESS J1825-137 and HESS J1826-130 display statistically preferred cut-offs and/or curvature in their spectral analysis (see Sect. 3.2 and H.E.S.S. Collaboration 2019, respectively). However they may still be able to partially contribute to the $\gtrsim 100 \mathrm{TeV}$ flux toward eHWC J1825134 (HAWC Collaboration 2020). In this regard, the preliminary results reported in Salesa Greus \& Casanova (2019) seem to support this scenario.

\section{Conclusions}

Finally, HESS J1826-130 is a particle accelerator in the Galactic plane capable of delivering $\mathrm{TeV}$ emission above several tens of $\mathrm{TeV}$. The deep observation campaign on the source reported in this paper provides several constraints to its spectral and morphological properties, although its origin is still uncertain. The presence of nearby SNRs found in the region and/or another yet unresolved accelerator, together with the gas density properties of the surroundings, could make hadronic interactions responsible for the observed emission. If protons are delivered with energies up to hundreds of TeV, HESS J1826-130 could point to the existence of a distinct population of $\mathrm{CR}$ sources that, along with the GC, significantly contribute to the Galactic CR flux around the knee feature. In a leptonic scenario, HESS J1826-130 has been proposed as the counterpart of the Eel Nebula powered by the pulsar PSR J1826-1256. Our observations reveal VHE emission above several tens of $\mathrm{TeV}$ that comes from the vicinity of the pulsar. Regarding the association of HESS J1826-130 with the recently discovered

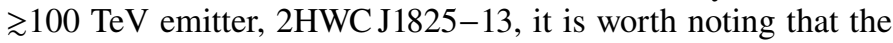

spectrum reported in Fig. 3 extends at least up to $\sim 43 \mathrm{TeV}$. Therefore, it could be possible that a fraction of the emission reported from eHWC J1825-134 could be partially contributed by HESS J1826-130 (see Salesa Greus \& Casanova 2019). On the other hand, HESS J1825-137 also displays VHE emission above tens of $\mathrm{TeV}$, albeit displaying a softer spectrum. Observations of the region, for example using the LHAASO observatory (Bai et al. 2019), and in the future with the Cherenkov Telescope Array (The CTA Consortium 2019) with its superior angular and energy resolution and sensitivity at energies above $10 \mathrm{TeV}$, will provide further insights into the origin of HESS J1826-130.

Acknowledgements. The support of the Namibian authorities and of the University of Namibia in facilitating the construction and operation of H.E.S.S. is gratefully acknowledged, as is the support by the German Ministry for Education and Research (BMBF), the Max Planck Society, the German Research Foundation (DFG), the Helmholtz Association, the Alexander von Humboldt Foundation, the French Ministry of Higher Education, Research and Innovation, the Centre National de la Recherche Scientifique (CNRS/IN2P3 and CNRS/INSU), the Commissariat à l'énergie atomique et aux énergies alternatives (CEA), the UK Science and Technology Facilities Council (STFC), the Knut and Alice Wallenberg Foundation, the National Science Centre, Poland grant no. DEC2017/27/B/ST9/02272, the South African Department of Science and Technology and National Research Foundation, the University of Namibia, the National Commission on Research, Science \& Technology of Namibia (NCRST), the Austrian Federal Ministry of Education, Science and Research and the Austrian Science Fund (FWF), the Australian Research Council (ARC), the Japan Society for the Promotion of Science and by the University of Amsterdam. We appreciate the excellent work of the technical support staff in Berlin, Zeuthen, Heidelberg, Palaiseau, Paris, Saclay, Tübingen and in Namibia in the construction and operation of the equipment. This work benefited from services provided by the H.E.S.S. Virtual Organisation, supported by the national resource providers of the EGI Federation.

\section{References}

Abdo, A. A., Ackermann, M., Ajello, M., et al. 2009, Science, 325, 840 Abeysekara, A. U., Albert, A., Alfaro, R., et al. 2017, ApJ, 843, 1 Abramowski, A., Aharonian, F., Ait Benkhali, F., et al. 2014, ApJ, 794, L1 Aharonian, F., Akhperjanian, A. G., Bazer-Bachi, A. R., et al. 2006a, A\&A, 457 899

Aharonian, F., Akhperjanian, A. G., Bazer-Bachi, A. R., et al. 2006b, ApJ, 636, 777

Aharonian, F., Akhperjanian, A. G., Bazer-Bachi, A. R., et al. 2006c, A\&A, 460, 365

Anderson, L. D., Wang, Y., Bihr, S., et al. 2017, A\&A, 605, A58

Bai, X., Bi, B. Y., Bi, X. J., et al. 2019, ArXiv e-prints [arXiv:1905. 02773]

Berge, D., Funk, S., \& Hinton, J. 2007, A\&A, 466, 1219

Bolmont, J., Corona, P., Gauron, P., et al. 2014, Nucl. Instrum. Meth., A761, 46

Brogan, C. L., Gelfand, J. D., Gaensler, B. M., Kassim, N. E., \& Lazio, T. J. W. 2006, ApJ, 639, L25

Deil, C., Zanin, R., Lefaucheur, J., et al. 2017, Int. Cosmic Ray Conf., 301, 766 de Naurois, M., \& Rolland, L. 2009, Astropart. Phys., 32, 231

Dickey, J. M., \& Lockman, F. J. 1990, ARA\&A, 28, 215

Duvidovich, L., Giacani, E., Castelletti, G., Petriella, A., \& Supán, L. 2019, A\&A, 623, A115

Gabici, S., Aharonian, F. A., \& Casanova, S. 2009, MNRAS, 396, 1629 Grondin, M. H., Funk, S., Lemoine-Goumard, M., et al. 2011, ApJ, 738, 42 Hartman, R. C., Bertsch, D. L., Bloom, S. D., et al. 1999, ApJS, 123, 79

HAWC Collaboration (Abeysekara, A. U., et al.) 2020, Phys. Rev. Lett. 124, 021102

H.E.S.S. Collaboration (Abramowski, A., et al.) 2016, Nature, 531, 476

H.E.S.S. Collaboration (Abdalla, H., et al.) 2018, A\&A, 612, A1

H.E.S.S. Collaboration (Abdalla, H., et al.) 2019, A\&A, 621, A116

Karpova, A. V., Zyuzin, D. A., \& Shibanov, Y. A. 2019, MNRAS, 487, 1964

Keogh, D. R. 2010, PhD thesis, University of Durham, UK

Knödlseder, J., Mayer, M., Deil, C., et al. 2016, A\&A, 593, A1

Lemiere, A., Terrier, R., \& Djannati-Atai, A. 2005, in 29th International Cosmic Ray Conference, 4, 105

Li, T.-P., \& Ma, Y.-Q. 1983, ApJ, 272, 317

MAGIC Collaboration (Acciari, V. A., et al.) 2020, A\&A, 642, A190

McClure-Griffiths, N. M., Dickey, J. M., Gaensler, B. M., et al. 2005, ApJS, 158, 178

Ohm, S., van Eldik, C., \& Egberts, K. 2009, Astropart. Phys., 31, 383 
Piron, F., Djannati-AtaÏ, A., Punch, M., et al. 2001, A\&A, 374, 895

Roberts, M. S. E., Romani, R. W., \& Kawai, N. 2001a, ApJS, 133, 451

Roberts, M. S. E., Romani, R. W., Kawai, N., Gaensler, B. M., \& Johnston, S. 2001b, Astrophys. Space Sci. Lib., 267, 135

Roberts, M. S. E., Gotthelf, E. V., Halpern, J. P., Brogan, C. L., \& Ransom, S. M. 2007, WE-Heraeus Seminar on Neutron Stars and Pulsars 40 years after the Discovery, ed. W. Becker, \& H. H. Huang, 24

Salesa Greus, F., \& Casanova, S. 2019, Int. Cosmic Ray Conf., 36, 781

Strong, A. W., Moskalenko, I. V., Reimer, O., Digel, S., \& Diehl, R. 2004, A\&A, 422, L47

The CTA Consortium 2019 https : //www . worldscientific . com/doi/pdf/ 10. $1142 / 10986$

Umemoto, T., Minamidani, T., Kuno, N., et al. 2017, PASJ, 69, 78

Vernetto, S., \& Lipari, P. 2016, Phys. Rev. D, 94, 063009

Voisin, F., Rowell, G., Burton, M. G., et al. 2016, MNRAS, 458, 2813

Wang, W. 2011, Res. A\&A, 11, 824

Zabalza, V. 2015, Int. Cosmic Ray Conf., 34, 922

Ziegler, A. 2018, PhD thesis, Friedrich-Alexander-Universität, ErlangenNürnberg, Germany

1 Centre for Space Research, North-West University, Potchefstroom 2520, South Africa

2 Laboratoire Leprince-Ringuet, école Polytechnique, CNRS, Institut Polytechnique de Paris, 91128 Palaiseau, France

3 Dublin Institute for Advanced Studies, 31 Fitzwilliam Place, Dublin 2, Ireland

4 Max-Planck-Institut für Kernphysik, PO Box 103980, 69029 Heidelberg, Germany

5 High Energy Astrophysics Laboratory, RAU, 123 Hovsep Emin St Yerevan 0051, Armenia

6 Aix-Marseille Université, CNRS/IN2P3, CPPM, Marseille, France

7 Laboratoire d'Annecy de Physique des Particules, Univ. Grenoble Alpes, Univ. Savoie Mont Blanc, CNRS, LAPP, 74000 Annecy, France

8 University of Oxford, Department of Physics, Denys Wilkinson Building, Keble Road, Oxford OX1 3RH, UK

9 IRFU, CEA, Université Paris-Saclay, 91191 Gif-sur-Yvette, France

10 University of Namibia, Department of Physics, Private Bag 13301, Windhoek 10005, Namibia

11 Instytut Fizyki Jạdrowej PAN, ul. Radzikowskiego 152, 31-342 Kraków, Poland

12 DESY, 15738 Zeuthen, Germany

13 Obserwatorium Astronomiczne, Uniwersytet Jagielloński, ul. Orla 171, 30-244 Kraków, Poland

14 Department of Physics and Electrical Engineering, Linnaeus University, 35195 Växjö, Sweden

15 Institut für Astronomie und Astrophysik, Universität Tübingen, Sand 1, 72076 Tübingen, Germany

16 Laboratoire Univers et Théories, Observatoire de Paris, Université PSL, CNRS, Université de Paris, 92190 Meudon, France

17 Sorbonne Université, Université Paris Diderot, Sorbonne Paris Cité, CNRS/IN2P3, Laboratoire de Physique Nucléaire et de Hautes Energies, LPNHE, 4 Place Jussieu, 75252 Paris, France
18 GRAPPA, Anton Pannekoek Institute for Astronomy, University of Amsterdam, Science Park 904, 1098 XH Amsterdam, The Netherlands

19 Friedrich-Alexander-Universität Erlangen-Nürnberg, Erlangen Centre for Astroparticle Physics, Erwin-Rommel-Str. 1, 91058 Erlangen, Germany

20 Astronomical Observatory, The University of Warsaw, Al. Ujazdowskie 4, 00-478 Warsaw, Poland

21 School of Physics, University of the Witwatersrand, 1 Jan Smuts Avenue, Braamfontein, Johannesburg 2050, South Africa

22 Université Bordeaux, CNRS/IN2P3, Centre d'Études Nucléaires de Bordeaux Gradignan, 33175 Gradignan, France

23 School of Physical Sciences, University of Adelaide, Adelaide 5005, Australia

24 Universität Hamburg, Institut für Experimentalphysik, Luruper Chaussee 149, 22761 Hamburg, Germany

25 Université de Paris, CNRS, Astroparticule et Cosmologie, 75013 Paris, France

26 Department of Physics and Astronomy, The University of Leicester, University Road, Leicester, LE1 7RH, UK

27 Nicolaus Copernicus Astronomical Center, Polish Academy of Sciences, ul. Bartycka 18, 00-716 Warsaw, Poland

28 Institut für Physik und Astronomie, Universität Potsdam, Karl-Liebknecht-Strasse 24/25, 14476 Potsdam, Germany

29 Laboratoire Univers et Particules de Montpellier, Université Montpellier, CNRS/IN2P3, CC 72, Place Eugène Bataillon, 34095 Montpellier Cedex 5, France

30 Landessternwarte, Universität Heidelberg, Königstuhl, D 69117 Heidelberg, Germany

31 Institut für Physik, Humboldt-Universität zu Berlin, Newtonstr. 15, 12489 Berlin, Germany

32 Institut für Astro- und Teilchenphysik, Leopold-FranzensUniversität Innsbruck, 6020 Innsbruck, Austria

33 Department of Physics, Rikkyo University, 3-34-1 Nishi-Ikebukuro, Toshima-ku, Tokyo 171-8501, Japan

34 Institute of Astronomy, Faculty of Physics, Astronomy and Informatics, Nicolaus Copernicus University, Grudziadzka 5, 87-100 Torun, Poland

35 Department of Physics, University of the Free State, PO Box 339, Bloemfontein 9300, South Africa

36 Department of Physics, The University of Tokyo, 7-3-1 Hongo, Bunkyo-ku, Tokyo 113-0033, Japan

37 Yerevan Physics Institute, 2 Alikhanian Brothers St., 375036 Yerevan, Armenia

38 Kavli Institute for the Physics and Mathematics of the Universe (WPI), The University of Tokyo Institutes for Advanced Study (UTIAS), The University of Tokyo, 5-1-5 Kashiwa-no-Ha, Kashiwa, Chiba, 277-8583, Japan

39 Now at Physik Institut, Universität Zürich, Winterthurerstrasse 190, 8057 Zürich, Switzerland

40 Now at Institut de Ciències del Cosmos (ICC UB), Universitat de Barcelona (IEEC-UB), Martí Franquès 1, 08028 Barcelona, Spain 